\title{
LABORATORY TESTING OF THE LEICA AT401 LASER TRACKER
}

\author{
FILIP DVOŘÁČEK ${ }^{a, b}$ \\ ${ }^{a}$ Czech Technical University in Prague, Faculty of Civil Engineering, Department of Special Geodesy, \\ Thákurova 7, 16629 Prague, Czech Republic \\ ${ }^{b}$ Research Institute of Geodesy, Topography and Cartography, Ústecká 98, 25066 Zdiby \\ correspondence: filip.dvoracek@fsv.cvut.cz
}

Abstract. This paper describes laboratory tests on a Leica AT401laser tracker. As the newer Leica AT402 model also uses the same firmware package, most of the results should also be valid for this device. First, we present the instrument's firmware errors and the software used for testing. The ASME B89.4.19-2006 standard for testing laser trackers is briefly presented. The warm-up effect of the instrument is inspected with respect to both angle measurement and distance measurement. The absolute distance meter (ADM) is compared with a laboratory interferometer on a 30-meter long rail and also on a bench with automated movement of the carriage of the reflector. A time series of measurements for determining the additive constant is evaluated. A simple test of the stability of the distance measurement in field conditions is introduced. Most of the tests were carried out at the Research Institute of Geodesy, Topography and Cartography (RIGTC) and at the Faculty of Civil Engineering (FCE) of the Czech Technical University in Prague (CTU).

KEYwORDS: laser tracker; Leica AT401; laboratory testing; firmware error; length metrology.

\section{INTRODUCTION}

The Leica AT401 laser tracker that is employed in all the tests reported on here is owned by the Department of Metrology and Engineering Geodesy of the Research Institute of Geodesy, Topography and Cartography (RIGTC). The primary use of the instrument is for calibrating the Koštice Czech State Long Distances Measuring Standard [1]. The Hvězda National Geodetic Baseline has also recently been measured. The tracker is also employed in other suitable calibration tasks for RIGTC's clients.

It is natural for a metrological laboratory to want to know the precision and the accuracy of a device that it uses. If the device is used as a calibration standard (an etalon), its accuracy has to be regularly proved to a higher authority during laboratory inspections. For AT401, the use of an authorized service only (e.g. Hexagon Metrology) would be an excessively expensive solution. Internal testing and compensation is therefore carried out. This paper summarises the findings from these procedures.

The paper does not cover all the tests of the geometrical errors of the instrument [2, and the main aim is not to make a thorough evaluation of the best measurement capability of AT401. The tests are focused on the use of the instrument for calibrating length baselines. The tests could be performed in most laboratories, as they do not require any special equipment. The instrument was tried out to see if it meets the requirements for specific tasks, and its accuracy parameters have been verified. It is expected that all findings presented here for Leica AT401 are also valid for the AT402 model. AT402 shares the same system software (firmware) as AT401 and, according to the manufacturer, only minor hardware upgrades have been made.

\section{ATCONTRol: User-Programmed CONTROLLING APPLICATION}

Leica Tracker Pilot software is supplied free-of-charge with the AT40x. It is capable of measuring and displaying fundamental geodetic quantities. Version 1.x did not allow data saving at all. Version 2.x allows some saving, but only manually and with a potential risk of data loss. It is therefore considered that Tracker Pilot is not fully specified for use in extended measurement tasks, but can be used rather for demonstrating the capabilities of the instrument. The main aim of the software is to mediate firmware updating, to administer the reflector and compensation file, and perform field and compensation test procedures.

Commercial software solutions for Leica AT40x have several shortcomings if they are used by surveyors. They are primarily focused on industrial metrology and coordinate measurements. They sometimes do not offer the possibility to save basic geodetic quantities (such as angles and distances), and do not provide basic surveying operations with the instrument (repeated measurements, two-face measurements, automation). In addition, they are very expensive to use.

ATControl [3] (Fig. 1) is a Matlab Mathworks application that has been programmed by the author of this paper. It is specifically designed for use in geodesy and for laboratory testing, and it enables all 


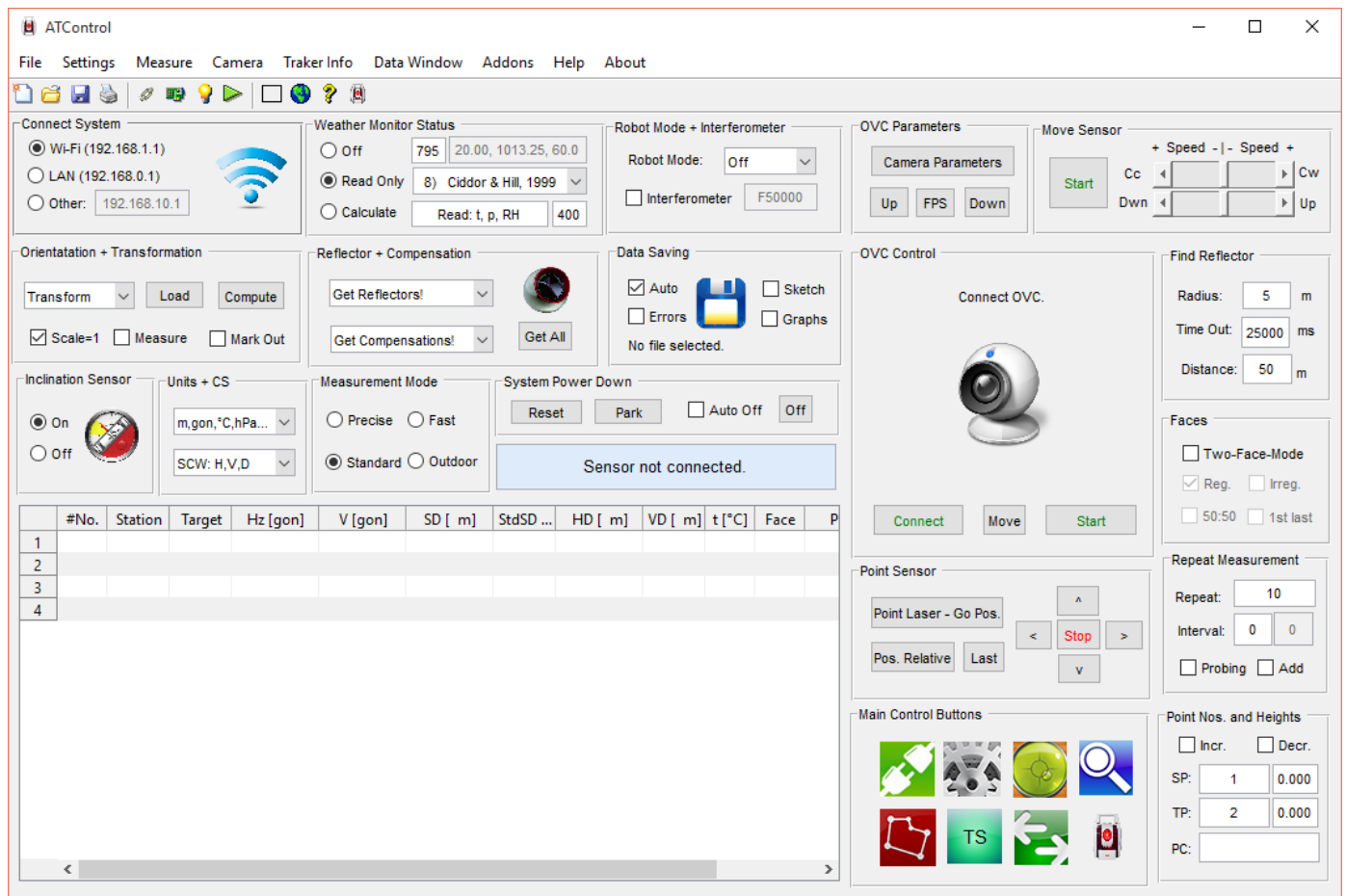

FIgURE 1. The ATControl v. 3.4 user-programmed controlling application.

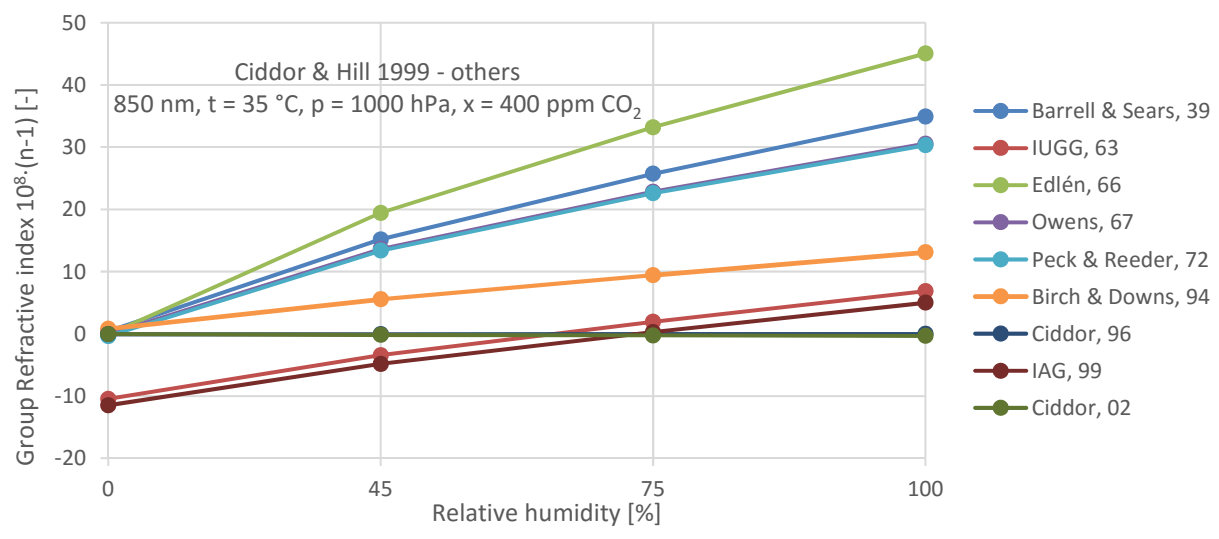

FiguRE 2. Relative analyses of 9 models for computing the group refractive index of air.

possible measured data to be saved, along with other evaluated values. ATControl is capable of correcting most of the errors described in Section 3. The application consists of over 30 .m files and over 4000 lines of source code in the main script. An up-to-date Matlab Mathworks or Matlab Runtime environment is required to run the compiled executable file. The Object Model (COM) Active Template Library (ATL) component, provided by Leica [4], is used for placing commands to the instrument. The application has a synchronous interface with a few commands which are asynchronous by nature (e.g. querying reflectors and compensations, obtaining transformation points, error reporting).

ATControl ha the following properties:

- offers 11 different refraction procedures,

- corrects AT40x system software errors,
- transforms the coordinate system,

- makes automated measurements with the FCE's interferometer,

- processes data from AT40x and Ahlborn Almemo Dataloggers,

- provides an overview camera (OVC) and image-click pointing of the sensor,

- makes automated sequence measurements of multiple points,

- has advanced two-face measurement modes.

All tests presented in this paper were carried out using the ATControl application, with the exception of monitoring the additive constant, which was performed using several different versions of the Tracker Pilot software, because the procedure is pre-designed by the manufacturer. 


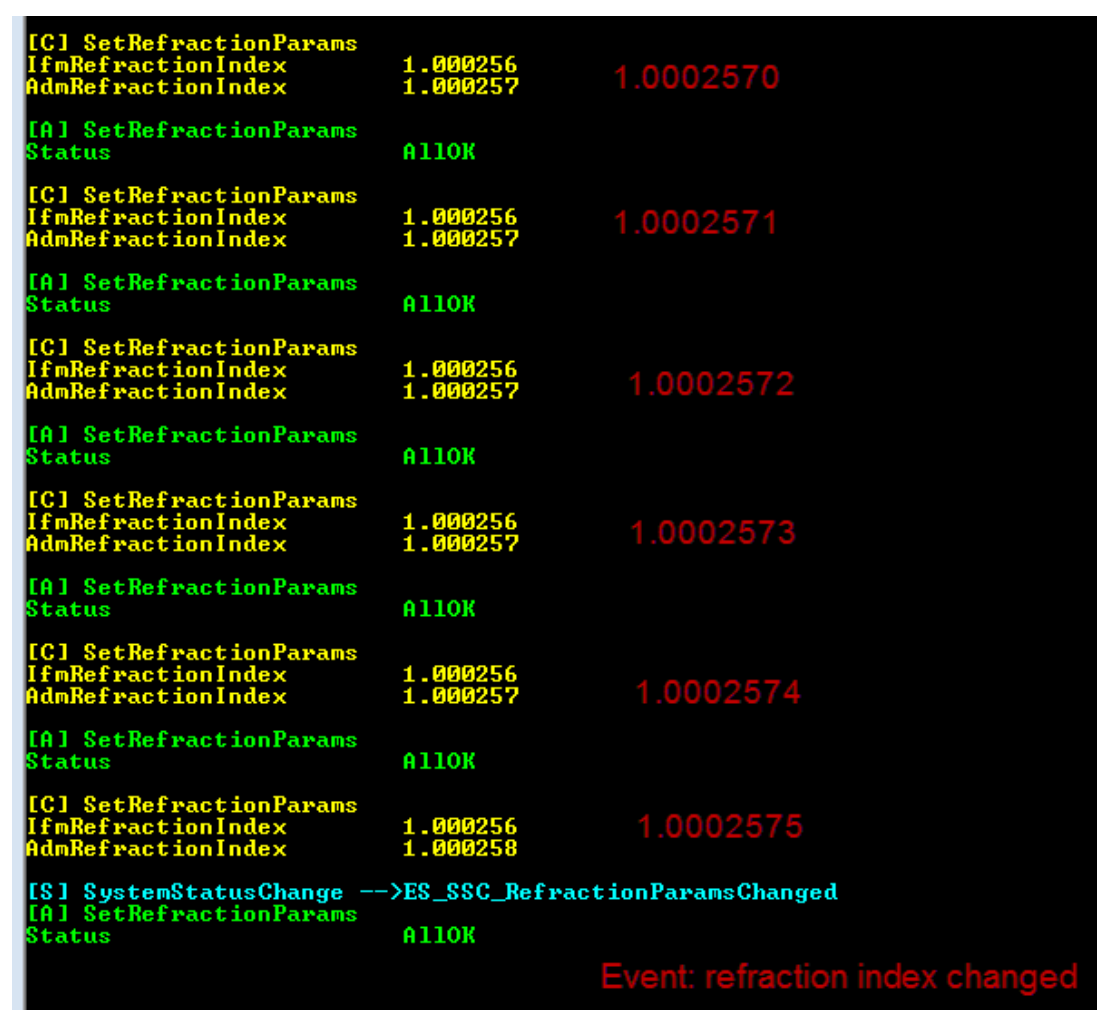

FiguRE 3. A print screen from TPIAnalyzer v. 2.2.9, provided by Leica's support.

\section{SySTEM SOFTWARE (FIRMWARE) ERRORS}

Errors were discovered in the v. 2.0.0.5053 system software, and have even proved to be present in later versions [5. No information concerning reported errors in firmware's descriptions has been observed.

\subsection{THE REFRACTIVE MODEL}

A document [6] describing the computation procedure for the group refractive index of air was obtained from Leica on 5th May 2013. According to the document obtained from Leica and also as observed in practical tests, AT401 uses equations derived from Edlén's formula 7 by default. It can differ by as much as $0.5 \mathrm{ppm}$ (Fig. 2] 8] from the Ciddor \& Hill procedure (1996 [9], 1999 [10]), which was recommended by the resolution of the IAG (International Geodetic Association) in Birmingham in 1999 [1]. Although these default formulas can be overruled by a user-programmed procedure, it is unlikely that many programmers would use this option.

\subsection{The Wavelength of the ADM}

The document from Leica states 6 that AT401 operates with a wavelength of $795 \mathrm{~nm}$. In the instrument's user manual [12], $780 \mathrm{~nm}$ is declared. Practical tests and discussions with Leica's support team showed that AT401 operates physically with the $795 \mathrm{~nm}$ wavelength laser beam, but that the instrument performs the computations with an improper wavelength of $780 \mathrm{~nm}$. Therefore, according to an error analysis of the equations listed below, there is a systematic distance-independent error of about $0.3 \mathrm{ppm}$ in the refractive index of air.

$$
\begin{aligned}
& N_{G r, p p m} \approx A P\left(\frac{1+10^{-6}(0.613-0.010 T)}{1+0.0036610 T}\right)-B R 10^{\frac{7.5 T}{T+237.3}+0.6609}, \\
& N_{G r} \approx 1+\frac{N_{G r, p p m}}{1000000} .
\end{aligned}
$$

\subsection{IMPROPER UPDATES OF THE GROUP REFRACTIVE INDEX OF AIR}

The error of the improper updates of the refractive indices is the most complicated error, and it is hard to discover. For some unexplained reason, the AT401 tracker neglects changes in the refractive index of air up to $0.5 \mathrm{ppm}$ (Fig. 3). Even if new atmospheric parameters are obtained from AT Controller 400 (ATC400) meteostation or if a newly-given user-computed refractive index is inserted, the emScon server does not update the value in its internal memory. As a consequence, the measured lengths are frequently corrected with an outdated value of the refractive index, sometimes even several hours old. This causes step changes of the measured distance (Fig. 5).

\subsection{IMPACTS OF AT40X FIRMWARE ERRORS}

A summary of the discovered errors has been made in order to demonstrate that the errors are significant and should be taken into account by all current and potential users. shows how the errors may effect measurements with AT40x in ordinary conditions - in the 


\begin{tabular}{lrrrrrr}
\hline \multirow{2}{*}{ Error } & \multicolumn{2}{c}{$\begin{array}{c}\text { Max. error } \\
(160 \mathrm{~m})\end{array}$} & \multicolumn{3}{c}{ Common error } \\
\cline { 2 - 7 } & \multicolumn{2}{c}{} & & \multicolumn{2}{c}{ Laboratory } & \multicolumn{2}{c}{ Outdoor } \\
\cline { 2 - 7 } & Min & Max & Min & Max & Min & Max \\
\hline Refractive model & 2 & 102 & 2 & 4 & 2 & 51 \\
Wavelength & -50 & -43 & -8 & -8 & -45 & -43 \\
Updating ref. index & -80 & 80 & -8 & 8 & -80 & 80 \\
\hline All togeather & -128 & 139 & -14 & 3 & -123 & 88 \\
\hline
\end{tabular}

TABLE 1. Impacts of the AT40x firmware errors $[\mu \mathrm{m}]$.

laboratory and outdoors. The impacts of the errors depend on the ambient atmospheric conditions and their gradients, and also on the distance to the target point. Both extremes (Min, Max) of these error intervals are evaluated in Table1 The maximum error is derived as the maximum possible influence for the working range of the whole instrument $\left(\langle 0 ; 160\rangle \mathrm{m}\right.$ distance, $\langle 0 ; 40\rangle^{\circ} \mathrm{C}$ temperature, $\langle 500 ; 1100\rangle \mathrm{hPa}$ pressure, $\langle 0 ; 95\rangle \%$ relative humidity). In laboratory conditions, stability of temperature $\pm 0.25^{\circ} \mathrm{C}$ at $20^{\circ} \mathrm{C}$ and $30 \mathrm{~m}$ length are assumed. A reduced temperature range of $\langle 0 ; 30\rangle^{\circ} \mathrm{C}$ is used for the outdoor evaluation. Note that an error in the group refractive index of air causes an error of about the same amount in the measured distance $(\mathrm{km})$. Each of the issues described above in itself potentially exceeds the manufacturer's specification of the accuracy of the distance measurement $(5 \mu \mathrm{m})[13$.

\section{Manufacturer's instrument TESTS AND STANDARDIZATION}

When Leica produces a laser tracker and hands it over to the customer, an initial quality certificate is supplied, in accordance with DIN 55350-18-4.2.2. The instrument's check consists of 4 different tests, which users can study before trying to reproduce the procedure. The manufacturer also defines procedures (Field, Sensor and Compensations) that can be utilized for checking whether the instrument works as it is supposed to. If the stated parameters are exceeded, compensation can be applied. Concerning the geometries of the tests, they can be performed at the RIGTC laboratory, or wherever the atmospheric conditions are sufficiently stable. For the Scale Bar Check, it is of course necessary to have a calibrated scale bar.

ASME B89.4.19-2006 Performance Evaluation of Laser-Based Spherical Coordinate Measurement Systems [14 is the well-known first standard for laser trackers. Unfortunately, the instrument tests are focused on the use of the instrument in industrial metrology rather than in surveying. The ADM ranging test described in subsection 6.5) is to be carried out with reference lengths established by the laboratory interferometer. The four measured lengths should be approximately 18, 36, 54 and $72 \%$ of the instrument's working range. The test cannot be performed for the
$160 \mathrm{~m}$ working range of AT401. The test is very simple to evaluate, because it consists of a comparison of differences within the maximum permissible error (MPE). No compensation of the errors is discussed or applied. A variation of the ADM scale test has been designed and performed at the RIGTC. Tests of laser trackers in compliance with ASME B89.4.19 have been studied at the National Institute of Standards and Technology [15], [16]).

The ISO/FDIS 10360-10 standard (Geometrical product specifications (GPS) - Acceptance and reverification tests for coordinate measuring systems (CMS) - Part 10: Laser trackers for measuring point-to-point distances.) is still under development, and is currently in the approval stage.

\section{Performance tests}

\subsection{Manufacturer's ADM CHECK/COMPENSATION}

During the ADM check, two target points are situated 4 meters apart. The additive constant can be computed from the distances between the target points, measured from the inside and the outside of the short baseline. A classic geodetic principle, where changing target points with station points is needed, would not produce the required accuracy. In the past, the geometry (from $3 \mathrm{~m}$ to $4 \mathrm{~m}$ ), the methodology (3 instead of 2 station point measurements) and also the tolerance interval (from 10 to $5 \mu \mathrm{m}$ ) of the ADM check were changed by the manufacturer. This obviously causes some difficulties for users. RIGTC had prepared a console with tribachs for quick setting of targets and the instrument in the right positions, but after the geometry of the test had been changed, it was no longer useful. Probably in order to check the stability of the points and the environment, a further measurement from the middle of the targets was added at the end of the procedure. When the tolerance interval was decreased from 10 to $5 \mu \mathrm{m}$, it was a step in the right direction. The additive constant can be determined even with about $1 \mu \mathrm{m}$ standard deviation by this test, so a $10 \mu \mathrm{m}$ allowance interval was excessively large. If users believe that the new determination of the constant is more accurate than the previous determination, it is always wise to allow Tracker Pilot to 


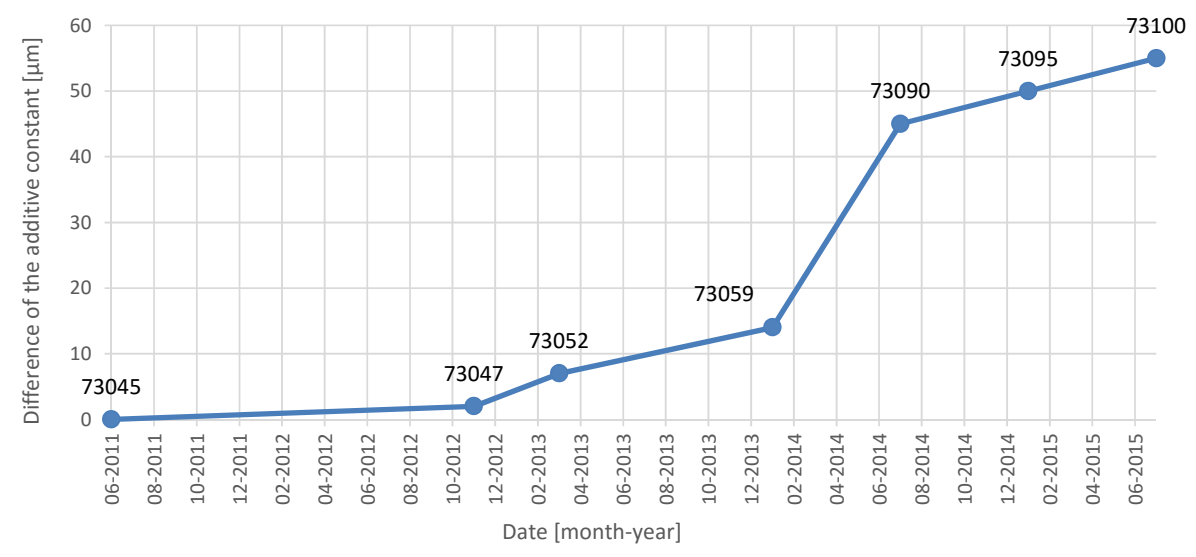

FIGURE 4. The time progress of the additive constant (AT401 S/N $390740+$ RRR 1.5in S/N 8058).

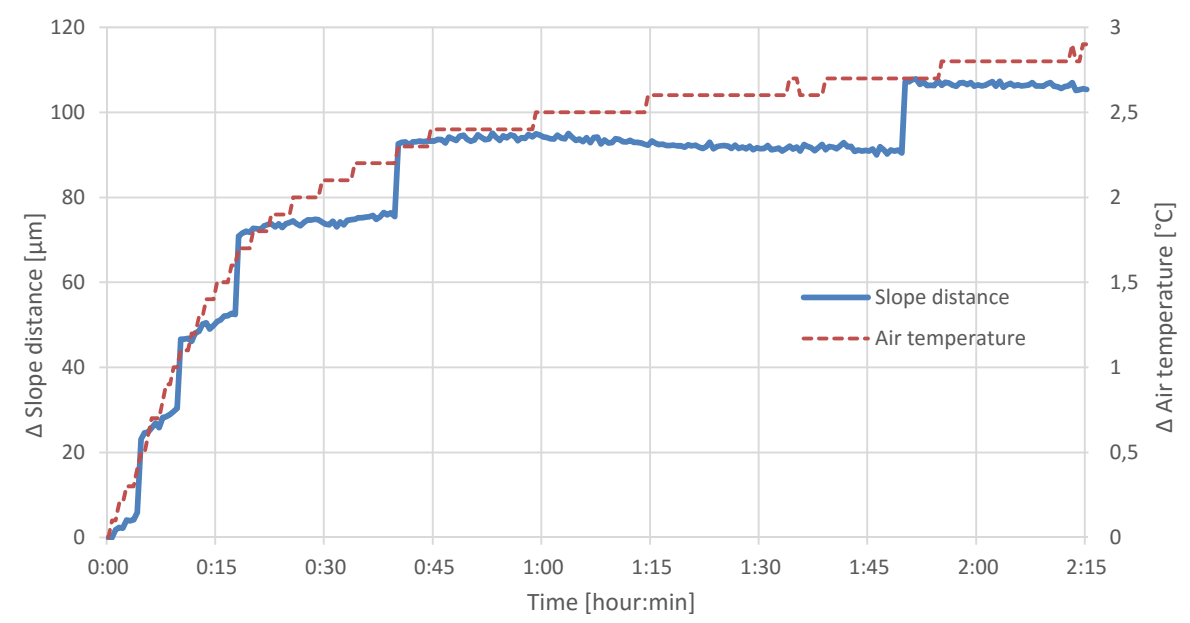

Figure 5. Testing the internal temperature sensor of the Leica ATC400 controller (length $31 \mathrm{~m}$ ).

compute the compensation, even if $100 \%$ tolerance was not achieved.

It is necessary to decide how often the additive constant should be checked/compensated. The choice should be made with respect to the required accuracy and the expected change of the constant. When a long distance comprises several shorter distances, the error of the additive constant is involved multiple times in the result. This the case when field length baselines are calibrated, where even a $10 \mu \mathrm{m}$ error of the additive constant can easily cause an error in the length of the baseline of more than $0.1 \mathrm{~mm}$. Of course, the progress of the additive constant differs from one instrument to another. The graph below (Fig. 4) demonstrates that the differences of the additive constant are not always random, and due to ageing of the instrument they do not oscillate around zero. The values are subjected to a trend, and a difference of $55 \mu \mathrm{m}$ is significant for many measurement tasks.

\subsection{Testing ATC400 meteostation}

The ATC400 controller is equipped with an integrated meteostation capable of measuring air temperature, atmospheric pressure and relative humidity. In addi- tion, the Leica NTC S2 external temperature sensor can be connected for measuring the air temperature and/or the object temperature. The refresh rate of all values is set to $20 \mathrm{~s}$ by the manufacturer. Unfortunately, this value cannot be changed by the user. External NTC S2, atmospheric pressure and humidity sensors were compared with RIGTC's the calibrated devices, and both met their accuracy specifications without employing additional corrections. Ahlborn NTC FNA32L100, a temperature sensor with similar specifications to NTC S2, was chosen for use at the target point sensor during outdoor measurements.

Not all users read the entire instrument manual [12. If they did, they would find the statement that: "To achieve the highest possible measurement accuracy it is recommended to determine the ambient temperature using the provided external temperature sensor. Using the internal temperature sensor can lead to erroneous measurements of $4-5$ ppm!" Because the controller is moisture and dust resistant (IP54 certification), the internal temperature sensor is covered, and the electronics inside ATC400 unavoidably heats the sensor. The incorrect temperature readings 


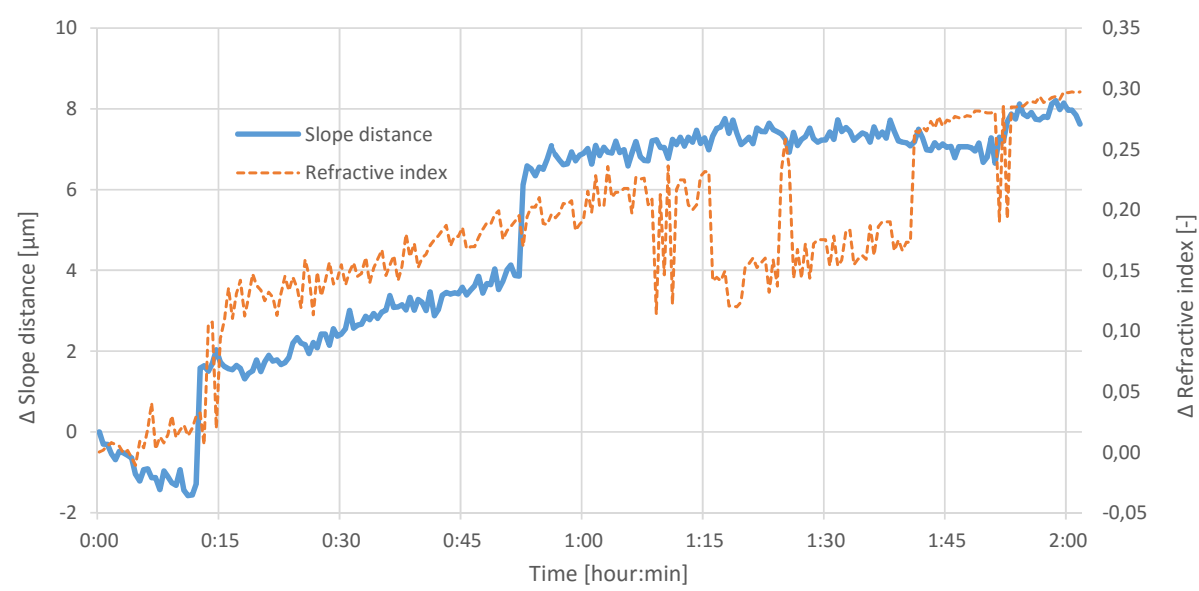

Figure 6. Monitoring a distance on a $2 \mathrm{~m}$ baseline during warm-up of Leica AT401.

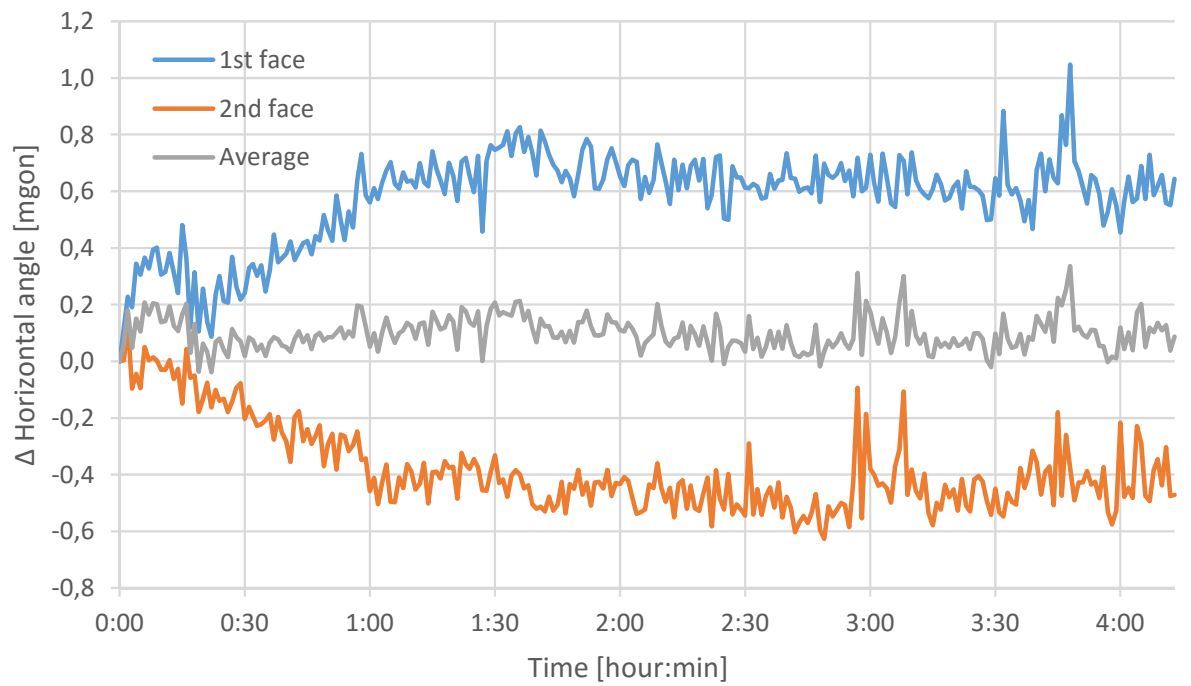

Figure 7. Monitoring horizontal angles on a $5 \mathrm{~m}$ baseline during warm-up of Leica AT401.

cause errors in the computed relative humidity and subsequently also in the distances corrected by the refractive indices of air.

Air temperatures and distances were compared on the $31 \mathrm{~m}$ stationary baseline (Fig. 5). Inside the RIGTC laboratory, the air temperature was stable within a couple of tenths of $1^{\circ} \mathrm{C}$ throughout the test lasting $2 \mathrm{~h} 15 \mathrm{~min}$. The temperature obtained from the internal temperature sensor rose by almost $3{ }^{\circ} \mathrm{C}$, and the measured slope distance therefore also changed proportionally. Noticeable step changes in the measured distance were caused by firmware errors in one of the Leica AT40x instruments: improper updates of the refractive index of the air (Subsection 3.3).

\subsection{THE WARM-UP EFFECT FOR ANGLES AND DISTANCES}

The warm-up effect is a detectable phenomenon which occurs in Leica's laser trackers. This has nothing in common with the standard adaptation of the instrument to the ambient temperature. The manufacturer has provided additional information for the author of the paper. The major cause of the warm-up effect is the inclination sensor in an oil bath. It suffers from heating of the instrument, and its offset therefore changes significantly. The zero position of the inclination sensor can be set by performing the initialization procedure. The manufacturer recommends that re-initialization should be performed every 30 minutes during the first 2 hours of measurements.

The warm-up effect was previously studied at RIGTC in Prague 17 in 2013. The stated result was that the sensor has to be turned on at least 2 hours before measurements are made. Unfortunately, the outcomes of the tests are strongly affected by using the internal temperature sensor instead of the external sensor. Even if the graph with the temperature progress is presented, no comment about the initial rise in temperature is given. The unreal change of the refractive index of air caused by the incorrect temperature observation devalued the distance measurement results. 


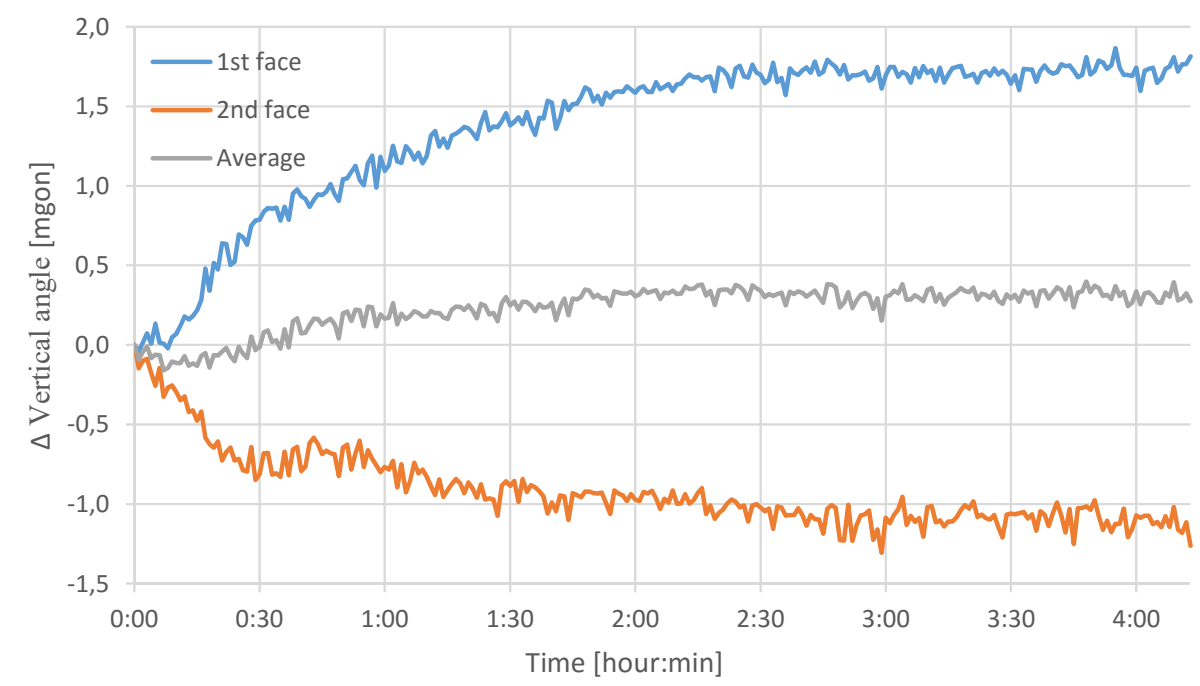

FIGURE 8. Monitoring vertical angles on a $5 \mathrm{~m}$ baseline during warm-up of Leica AT401.

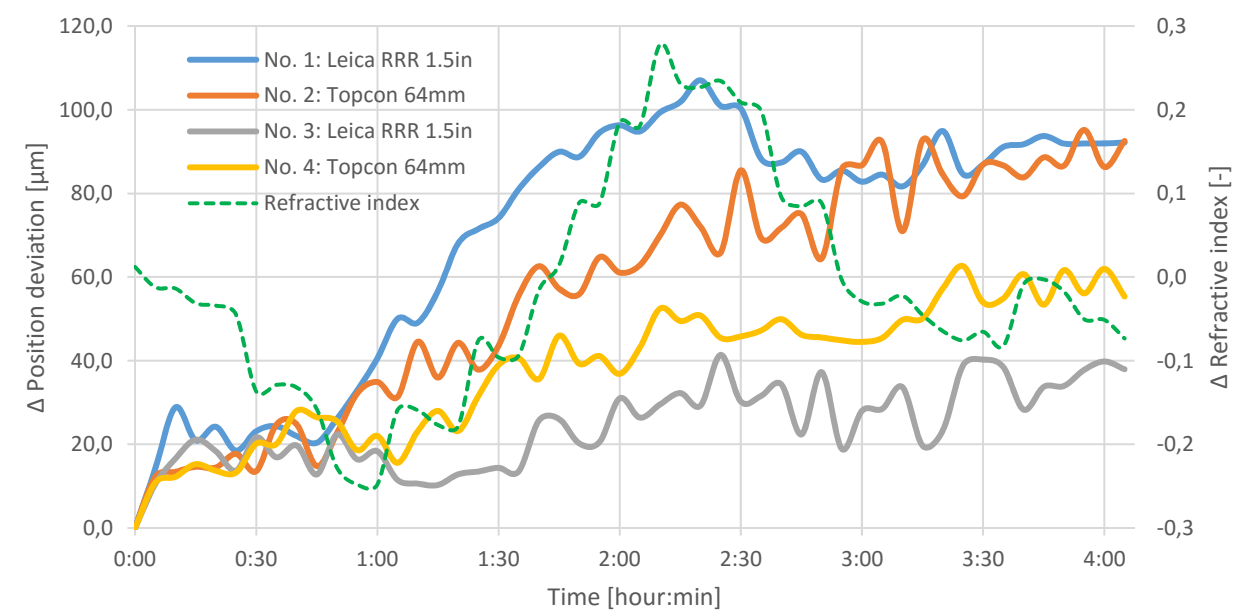

FiguRE 9. Four-hour monitoring of four points in a laboratory with Leica AT401.

Extended general warm-up effect testing has been performed by the National Accelerator Laboratory (SLAC) of Stanford University [18. One of the first firmware versions (1.0.373.0) was installed in Leica AT401. The least squares adjustment was used for evaluation translation and rotation of the centre, the scale factor and the distance offset of the instrument, and for changing the alignment of the axis.

The author of this paper ran 7 tests to monitor the stability of the measured values. The distances ranged between $2 \mathrm{~m}$ and $31 \mathrm{~m}$, the measurement intervals ranged between $30 \mathrm{~s}$ and $75 \mathrm{~s}$, and measurement time ranged between $2 \mathrm{~h}$ and $17 \mathrm{~h}$. Several interesting findings were obtained, and the main results are presented below. Problems with the stable baseline and with stable environmental conditions had to be dealt with at the RIGTC laboratory. The most reliable tests were therefore done with shorter distances.

Distances are influenced by the warm-up effect at a relatively low level (Fig. 6). For a 2-meter-long distance, changes in the refractive index play a minor role. Nevertheless, they have been included in the graph for demonstration purposes. Altogether, about $10 \mu \mathrm{m}$ offset can be detected, which is in congruence with [18. A step change with a difference of a couple of micrometres is very often detected during the first 2 hours. Two-face measurement does not make a significant difference. Tests for long distances showed no proven change in scale.

As expected, it is important to take two-face measurements into account when monitoring horizontal and vertical angles. A baseline of about 5 meters is more suitable for testing, because measurement accuracy decreases for angle measurements of short distances. Even if the 1st and 2nd face angles have obvious contradictory trends, the average values of the two faces stay within much more reasonable limits (Figs. 7 and 8). Two-face-measurements allow observations to begin almost immediately for most ordinary surveying tasks. While it takes from about one and a half to two hours to stabilize the horizontal angle, it takes about 3 to 4 hours to settle the vertical angle. 


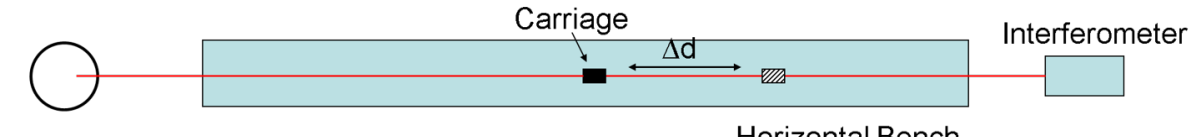

Laser Tracker

Horizontal Bench

Figure 10. Test settings: a comparison between Leica AT401 and Interferometer HP 5519A [18].
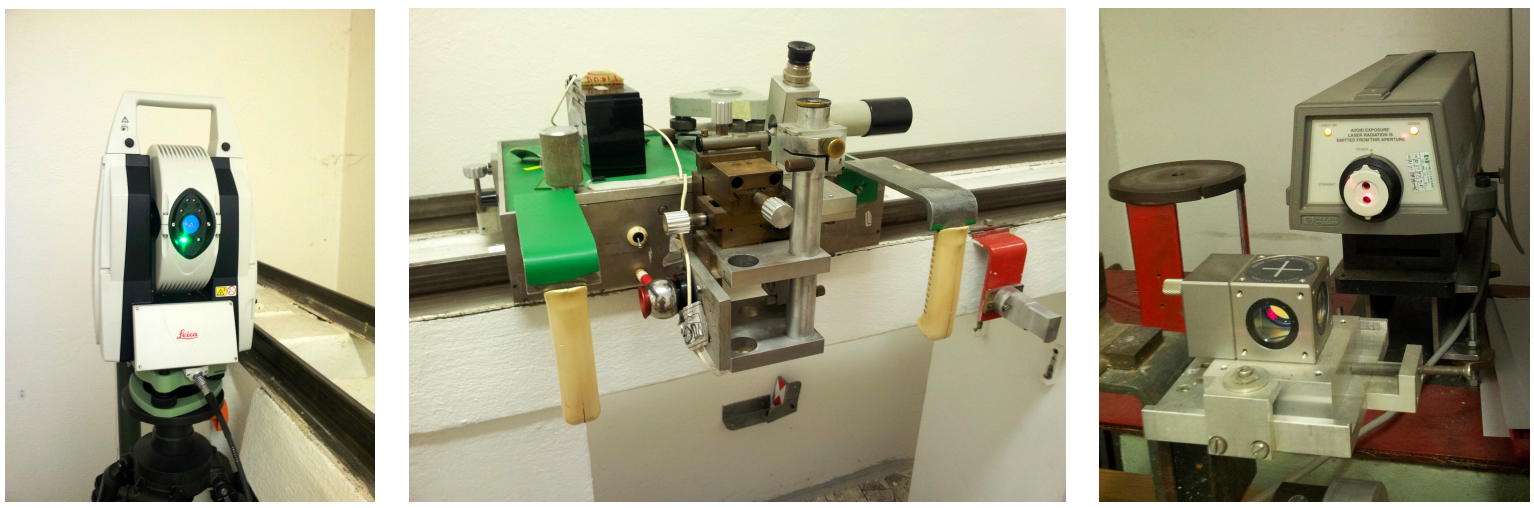

FiguRE 11. Left: Leica AT401; Centre: The interferometer carriage; Right: Interferometer HP 5519A.

In addition, the magnitude of the changes is about twice as much for the vertical angles as for the horizontal angles. This is in agreement with the finding reported in an SLAC paper [18] that the translation and the rotation of the centre of the instrument is much greater in the $\mathrm{Z}$ axis than in the $\mathrm{X}$ and $\mathrm{Y}$ axes.

\subsection{Continuous measurements IN A LABORATORY MICRONETWORK}

A micronetwork consisting of 4 points was established at the RIGTC laboratory. The 2 points mounted with Leica RRR 1.5in were planted on the higher wall, while 2 other points equipped with Topcon $64 \mathrm{~mm}$ prisms were located on the rail of the interferometer. All points were observed in two-face mode at 5 -minute intervals for more than 4 hours. The test was carried out for several purposes. The first aim was try out in action a mode of ATControl software for automated sequence measurements (robot-mode). As the laser tracker started working immediately after start-up, the second aim was to continue our research on the warm-up effect. The third aim was to evaluate whether measurements to a standard geodetic prism by Topcon differ in terms of repeatability from measurements to Leica RRR 1.5in The graph presented in Fig. 9 shows the position deviations $\left(p=\sqrt{\Delta x^{2}+\Delta y^{2}+\Delta y^{2}}\right)$ of the target points.

The robot mode of ATControl software worked well, and it is ready for use in appropriate monitoring tasks. The results for the precision of position determination of RRR and Topcon prisms are similar. In a different test, the usability of Leica mini prisms (such as GMP101) was verified. Of course, the centring of optical parts and the centring of mechanical parts are a qualitatively different matter, and this must always be taken into account. However, when multiple reflectors are needed and absolute positions of points can be omitted, standard prisms can be an adequate substitute for spherically mounted retroreflectors (SMR). The test was not considered reliable for further conclusions on warm-up effects. Although the RIGTC laboratory is located underground, the thermal expansivity of the building affects the measurements, and the resulting deviations have to be assigned partly to movements of the points.

\subsection{Checking the SCAle With A LABORATORY INTERFEROMETER}

The scale of distances measured with Leica AT401 cannot be checked without employing another instrument, e.g. a laser interferometer. The HP 5519A calibrated interferometer and a $30 \mathrm{~m}$ rail were used for this purpose at the RIGTC laboratory. According to the specifications $(U=Q[0.01 ; 0.2 L ; 0.2 \alpha L] \mu \mathrm{m})$, the accuracy of this interferometer is $3 \mu \mathrm{m}$ over the $30 \mathrm{~m}$ working range. All distances measured by the laser tracker and the interferometer were corrected using Ciddor [9] and Ciddor \& Hill [10] procedures approved by the International Association of Geodesy (IAG) [11. Naturally, both group refractive indices and phase refractive indices had to be considered. The geometry of the test (Fig. 10) was similar to the geometry used for the test performed at SLAC [18]. A slight vertical discrepancy of the instruments (Fig. 11), caused by restrictions in the laboratory, had to be eliminated numerically by measuring the vertical angles.

Although the RIGTC laboratory is equipped with air conditioning in the ceiling, there were some problems concerning the ambient atmosphere. The concept of the test, where the instruments are placed on opposite sides of the baseline, is extremely sensitive to refractive corrections. Initial test results showed different trends, which could not be assigned either to 


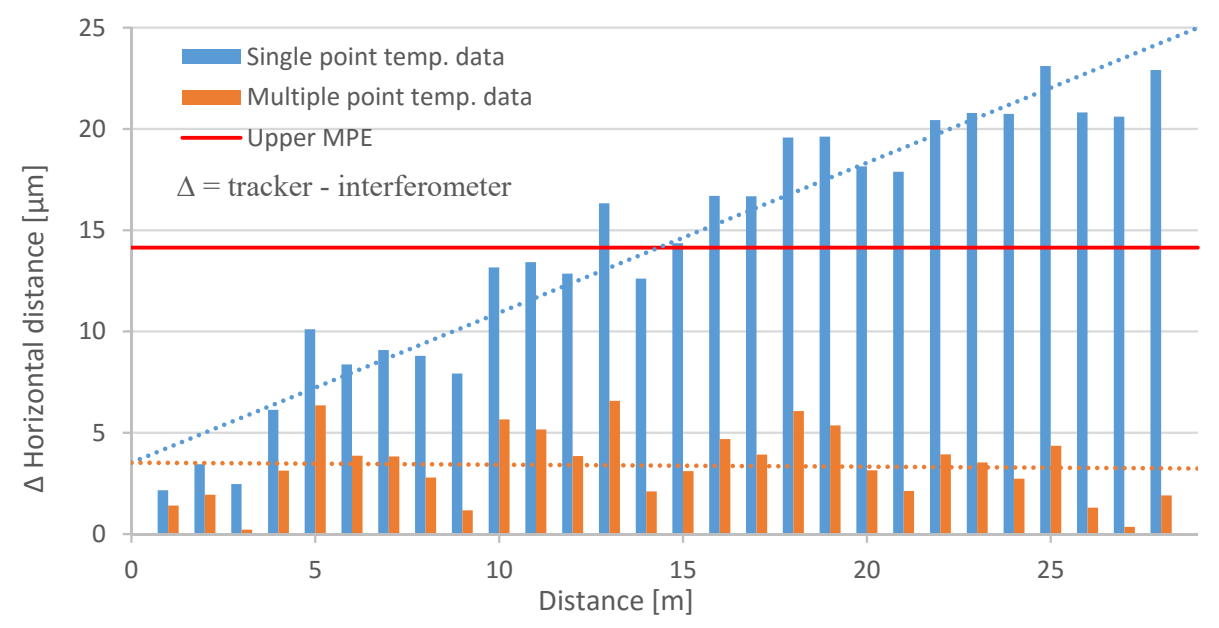

FIgURE 12. Checking the scale of distances of Leica AT401 with a laboratory interferometer.
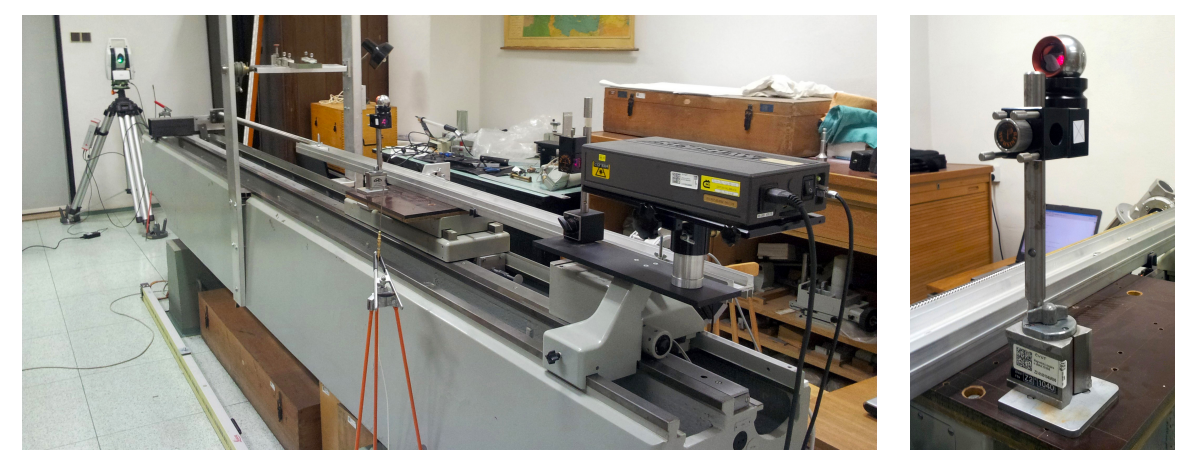

FiguRE 13. Left: Setup of the short periodic test; Right: The interferometer carriage with reflectors.

the laser tracker or to the interferometer with respect to their stated accuracy. The trends could be removed from the data only after adding multiple temperature sensors along the paths of the beams and evaluating the corrections of the distances manually. By this procedure, standard deviation of $1.7 \mu \mathrm{m}$ was achieved (Fig. 12). The offset on the Y-axes is not relevant for this test. The laser tracker passed the modified ASME B89.4.19-2006 ADM ranging test.

\subsection{LOOKING FOR SHORT PERIODIC ERRORS}

As Leica AT40x uses the advanced Kern ME5000 principle with adjustable frequencies [19, it was not anticipated that significant long or short periodic errors of the ADM would be found. Nevertheless, it was desirable to compare distance measurements with the interferometer on the short resolution basis. In order to obtain more reliable results, the tests were carried out in the laboratory of the department of geomatics at FCE CTU in Prague. A Renishaw ML10 interferometer, a short rail and a Microcon M1486 stepper motor were available. The automatic movement of the carriage was set to $2 \mathrm{~mm}$ resolution. Both the laser tracker and the interferometer were controlled by the ATControl application, which enabled synchronous measurements and data saving.

The very first manual tests with the HP 5519A in- terferometer at the RIGTC laboratory revealed some data discontinuity. It was also revealed in the automated test with Renishaw ML10 (Fig. 13). As differences of a couple of micrometres occur more or less randomly (Fig. 14), it has not been found out whether they are time-dependent or distance-dependent. Of course, they need not to be visually dependent on either of these factors. In fact, the repeatability of the ADM for short range is excellent, and can be considered better than the $5 \mu \mathrm{m}$ accuracy stated by the manufacturer. However, when the instrument is pushed to its limits, users should pay attention to the random data discontinuities.

\subsection{Continuous measurements in Field CONDITIONS}

Although laser trackers are mostly used indoors, RIGTC employs the AT401 during calibrations of field length baselines. Naturally, the capabilities of the instrument are limited by the harshness of the outdoor environment. At the Koštice baseline, two distances of about $76 \mathrm{~m}$ and $95 \mathrm{~m}$ were observed for more than 1 hour (Fig. 15). There was unfavourable sunny weather with temperatures of only about $3{ }^{\circ} \mathrm{Cduring}$ this test. It was intended to make the measurements at a temperature below $10^{\circ} \mathrm{C}$, because readings from the NTC sensor connected to AT400 could be ob- 


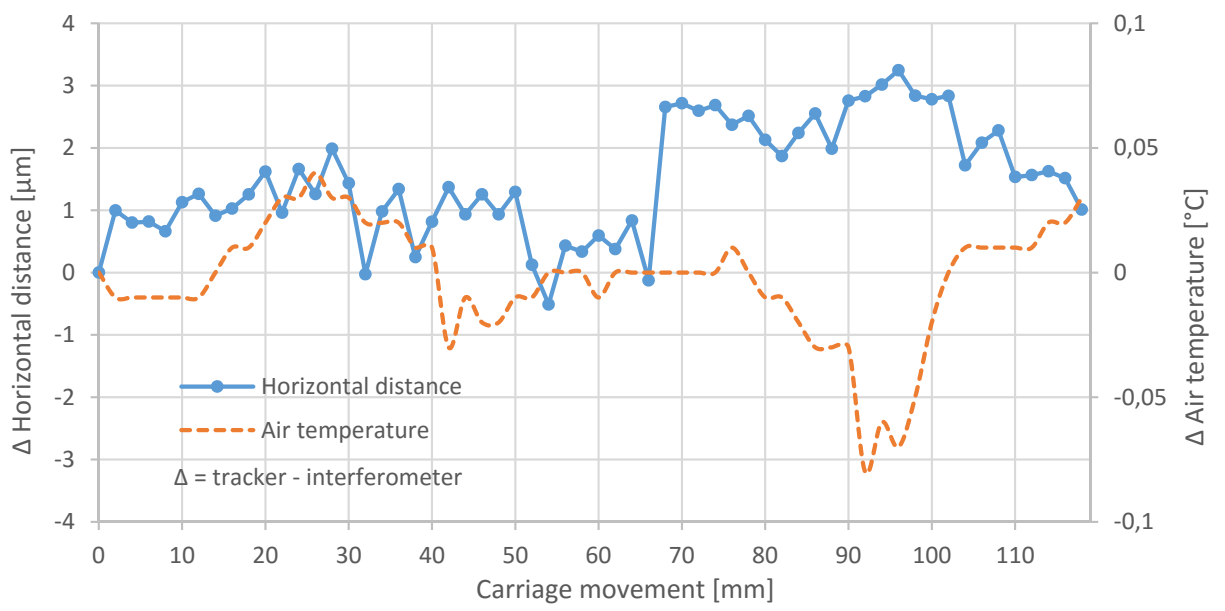

FiguRE 14. Looking for short periodic errors of Leica AT401 (2 mm resolution).

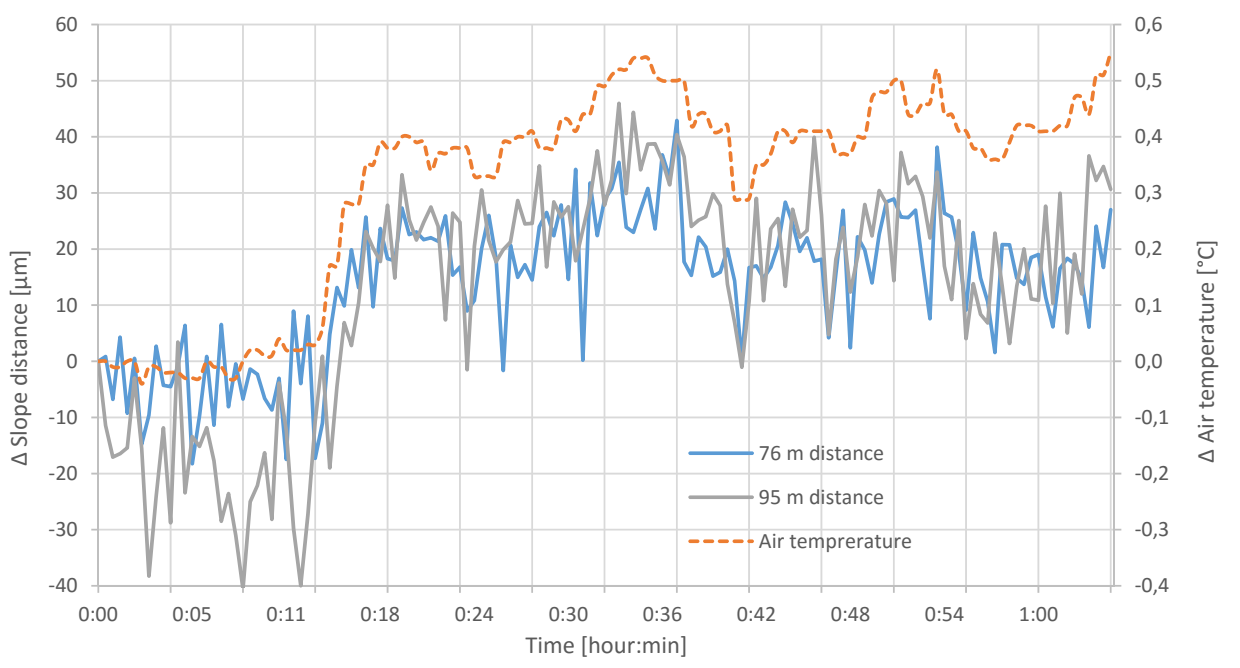

Figure 15. 1-hour continuous measurement with AT401 at the Koštice baseline.

tained to $0.01{ }^{\circ}$ Cresolution. Both monitored distances were verifiably affected by temperature observations, which were held only at the instrument's station point. Adding more temperature sensors along the beam path would have decreased this dependency. Even without the additional temperature meters, the standard deviation for the $76 \mathrm{~m}$ distance was $13 \mu \mathrm{m}$, and for the $95 \mathrm{~m}$ distance the standard deviation was $20 \mu \mathrm{m}$. Distances up to $180 \mathrm{~m}$ are observed at the Koštice Long Distances Measurement Standard.

\section{Conclusions}

The tested Leica AT401 generally fulfils the specifications declared by the manufacturer. However, there are some system software errors, which have been eliminated with the use of ATControl software. The issue with late updates of the group refractive index of air is the principle error. It degrades the measurements, because significant changes of the refractive index are omitted. When frequent re-initialization is required during a measurement routine, there is not much need to take care about the warm-up effect. However, the inclination sensor needs to be properly warmed up when a series of single-face measurements is to be performed. Along with SMRs, mini prisms such as Leica GMP101 and standard-size geodetic prisms are useful for measuring the relative movements of target points.

The progress of the additive constant should be continuously monitored, especially in cases where multiple distances are combined together. No scale correction had to be applied to the ADM, but the test settings with a laser interferometer are very sensitive to the ambient atmospheric parameters. This is of course also valid for long-range measurements in field conditions, where AT40x is very well usable. However, the temperature has to be observed at multiple points along the beam path, and the measurements need to be made under favourable weather conditions (stable, cloudy, night). Step changes of a couple of micrometres in a measured distance were detected during several different tests, but the magnitude of the error is within the manufacturer's accuracy specification. 


\section{REFERENCES}

[1] Lechner, J., Červinka, L., Kratochvíl, J., Umnov, I.: The Koštice New Czech State Etalon of Long Distances (in Czech). Research Institute of Geodesy, Topography and Cartography, 2007. http://www . vugtk.cz/odd25/kostice/geos.pdf [Sep. 2015].

[2] Zhu, J., et al.: Geometric error detection and calibration in laser trackers. Proc. of SPIE 9623. Bellingham, USA, 2015.

[3] Dvořáček, F.: Software: ATControl. http://k154.fsv.cvut.cz/ dvoracek/software.html [Sep. 2015].

[4] Leica Geosystems: EmScon 3.8: Leica Geosystems Laser Tracker Programming Interface - Programmers Manual. 2013. http://metrology.leica-geosystems . com/common/shared/downloads/inc/downloader .asp? id_0=20453\&submit_0=Download\&id_1=19781 [Sep. 2015].

[5] Dvořáček, F.: System Software Testing of Laser Tracker Leica AT401. Geoinformatics FCE CTU, 13(-), 2014, 49-57.

[6] Leica Geosystems: Formula for Calculating the Refractive Index of Ambient Air used for the Leica AT401 of Hexagon Metrology. 2013.

[7] Edlén, B.: The refractive index of air. Metrologia, en, 1966, 2, 71-80.

[8] Dvořáček, F.: Indirect Determination of Air Refractive Index for the First Velocity Correction of Electronic Distance Meters (Czech). Geodetický a kartografický obzor, 101, 2013, 253-266.

[9] Ciddor, P. E. (1996). Refractive index of air: New equations for the visible and near infrared. Applied Optics, 35(-), 1566-1572.
[10] Ciddor, P. E., Hill, R. J.: Refractive index of air. 2. Group index. Applied Optics, 38, 1999, 1663-1667.

[11] Rüeger, J. M.: Refractive Indices of Light, Infrared and Radio Waves in the Atmosphere. University of New South Wales, 2001.

[12] Leica Geosystems. Leica AT401 User Manual v.2.0. Switzerland, 2013.

[13] Leica Geosystems. Leica Absolute Tracker AT401: ASME B89.4.19-2006 Specifications. 2010. http: //www.geotech.sk/downloads/Totalne-stanice/ AT401/AT401_ASME_specifikacie_presnosti_en.pdf [Sep. 2015].

[14] The American Society of Mechanical Engineers: ASME 89.4.19-2006: Performance Evaluation of Laser-Based Spherical Coordinate Measurement Systems. New York, USA, 2006.

[15] Muralikrishnan, B., et al.: Performance Evaluation of Laser Trackers. Proc. PerMIS '08. New York, USA, 2008, 149-155.

[16] Muralikrishnan, B., et al. ASME B89.4.19 Performance Evaluation Tests and Geometric Misalignments in Laser Trackers. Journal of Research of the National Institute of Standards and Technology, 2009, 114:21-35.

[17] Hánek, P., Volkmann, M.: Testing the Leica AT-401 Absolute Tracker Laser Tracker (in Czech). Geodetický a kartografický obzor, 101(10), 2013, 229-234.

[18] Gasner, G., Ruland, R.: Instrument Tests with the New Leica AT401. Stanfort, CA, USA, 2011. http://www.slac.stanford.edu/cgi-wrap/getdoc/ slac-pub-14300.pdf [Sep. 2015].

[19] Bell, B.: The Use and Calibration of the Kern ME5000 Mekometer. Stanford, CA, USA, 1992. http://www.slac.stanford.edu/pubs/slacreports/ reports03/slac-r-403.pdf [Sep. 2015]. 